\title{
ON A CERTAIN INVARIANT OF A LOCALLY COMPACT GROUP
}

\author{
BY HORST LEPTIN
}

Communicated by E. Hewitt, April 28, 1966

Group here always means a locally compact Hausdorff group, subgroup means a closed subgroup. Let $G$ be a group, $H$ a subgroup and $G / H$ the locally compact homogeneous space of left cosets $\dot{x}=x H$. We denote by $\Re(G)[\Re(G / H)]$ the family of all compact subsets of $G[G / H]$. The group $G$ acts on $G / H$ in a natural way. If $X \subset G$ and $Y \subset G / H$, write $X Y$ for the set of all elements $x \dot{y}, x \in X, \dot{y} \in Y$. Now assume that $G / H$ has a nontrivial invariant positive measure $d \dot{x}$, e.g. the left invariant Haar measure, if $H$ is normal. For a measurable set $U$ in $G / H$ let $|U|$ or $|U|_{G / H}$ be its measure. Then we define:

$$
I(G / H)=\sup _{\boldsymbol{K} \in \mathscr{R}(G)} \inf _{\substack{U \in \mathscr{R}(G / H) \\|U|>0}} \frac{|K U|_{G / H}}{|U|_{G / H}} .
$$

Evidently $1 \leqq I(G / H) \leqq \infty$ and $1=I(G / H)$ if $G / H$ is compact. Let $E$ be the trivial subgroup of order one in $G$. We identify $G$ and $G / E$.

For a positive Radon measure $\mu$ and a Borel function $f$ on $G$, the convolution $\mu * f$ is defined as

$$
\mu * f(x)=\int_{G} f\left(y^{-1} x\right) d \mu(y) .
$$

If $\mathfrak{F}$ is a set of Borel functions, let $\mu * \mathfrak{F}$ be the set of all $\mu * f, f \in \mathfrak{F}$ (if this set is well defined). For $1 \leqq p \leqq \infty$, let $\Omega^{p}(G)$ be the usual $\mathfrak{R}^{p}$-space of the group $G$. If $\mu$ is a positive bounded Radon measure, then $\mu * \Re^{p}(G) \subset \mathbb{R}^{p}(G)$ for all $p \geqq 1$. In [2] I proved a partial converse of this fact, as follows.

Let $p>1$. If $\mu * \mathfrak{R}^{p}(G) \subset \mathbb{R}^{p}(G)$ and $I(G)<\infty$, then $\mu$ is bounded.

As I pointed out in [2], this implies the following.

Let $p>1$. If $\mathfrak{R}^{p}(G)$ is closed under convolution and $I(G)<\infty$, then $G$ is compact.

This latter statement, without the hypothesis $I(G)<\infty$, is the so called $R^{p}$-conjecture, stated and discussed by Żelazko, Rajagopalan and others [3], [4], [5], [6], [7].

The main result of this note is an inequality for $I(G)$, which implies the finiteness of $I(G)$ for a fairly large class of groups. Actually it reduces the problem of checking this finiteness to the case of simple Lie groups and finitely generated discrete groups. 
ThEOREM 1. Let $H$ be a subgroup of $G$. If the homogeneous space $G / H$ has a nontrivial positive invariant measure, then

$$
I(G) \leqq I(G / H) I(H) .
$$

We start with some definitions and lemmas. Let $d x, d \xi$ and $d \dot{x}$ be the (left) invariant measures in $G, H$ and $G / H$ respectively. For $f \in \mathfrak{R}^{1}(G), \dot{x} \in G / H$ let

$$
f(\dot{x})=\int_{H} f(x \xi) d \xi .
$$

The function $\dot{f}$ is well defined almost everywhere on $G / H$ and belongs to $\mathfrak{R}^{1}(G / H)$; furthermore

$$
\int_{G} f(x) d x=\int_{G / H}\left(\int_{H} f(x \xi) d \xi\right) d \dot{x}=\int_{G / H} f(\dot{x}) d \dot{x},
$$

(see e.g. $[1, \S 2$, no. 5$]$ ).

The image of a set $X \subset G$ in $G / H$ is denoted by $\dot{X}$, the characteristic function of a set $A$ by $\chi_{A}$.

Lemma 1. Let $K \in \Re(G), W \in \Re(H)$ and $Q=K^{-1} K \cap H \in \Re(H)$. Then

$$
|K W|_{G} \leqq|\dot{K}|_{G / H}|Q W|_{H} .
$$

Proof. For $\dot{x} \notin \dot{K}, x \in \dot{x}$ and $\xi \in H$ we have $x \xi \notin K W$, hence $\chi_{K W}(x \xi)=0, \dot{\chi}_{K W}(\dot{x})=0$. For $x \in K$ we have

$$
x^{-1} K W \cap H \subset K^{-1} K W \cap H=\left(K^{-1} K \cap H\right) W=Q W,
$$

hence $\chi_{K W}(x \xi)=\chi_{x^{-1} K W}(\xi) \leqq \chi_{Q W}(\xi)$ and

$$
\dot{\chi}_{K W}(\dot{x})=\int_{H} \chi_{K W}(x \xi) d \xi \leqq \int_{H} \chi_{Q W}(\xi) d \xi=|Q W|_{H} .
$$

Finally

$$
\begin{aligned}
|K W|_{G}=\int_{G} \chi_{K W} d x=\int_{G / H} \dot{\chi}_{K W} d \dot{x} & =\int_{K} \dot{\chi}_{K W} d \dot{x} \leqq|Q W|_{H} \int_{\dot{K}} d \dot{x} \\
& =|\dot{K}|_{G / H}|Q W|_{H .}
\end{aligned}
$$

Lemma 2. For $K \in \mathfrak{\Omega}(G)$ the following inequality holds:

$$
|\dot{K}|_{G / H} \leqq \inf _{W \in \Omega(H)} \frac{|K W|_{G}}{|W|_{H}} \leqq|\dot{K}|_{G / H} I(H) .
$$


Proof. The right part of the inequality is an immediate consequence from Lemma 1 . Now let $x \in K$ and $W \in \Re(H)$. Then $W \subset x^{-1} K W$, hence for $\xi \in H$ we have:

$$
\begin{aligned}
\chi_{K W}(x \xi) & =\chi_{x^{-1} K W}(\xi) \geqq \chi_{W}(\xi), \\
|K W|_{G} & =\int_{\dot{K}} \int_{H} \chi_{K W}(x \xi) d \xi d \dot{x} \geqq \int_{\dot{K}} \int_{H} \chi_{W}(\xi) d \xi=|\dot{K}|_{A / H}|W|_{H},
\end{aligned}
$$

which proves the left part of the inequality.

Now we can prove Theorem 1 . Take $K, U \in \Re(G)$ with $|U|_{G}>0$. For $\epsilon>0$, Lemma 2 implies the existence of a $W \in \Omega(H)$ with $|W|_{H}>0$ such that

$$
\begin{aligned}
|(K U) \cdot|_{G / H} & =|\dot{K} U|_{G / H} \leqq \frac{|K U W|_{\theta}}{|W|_{H}} \leqq|\dot{K} \dot{U}|_{G / H}(I(H)+\epsilon), \\
|\dot{U}|_{G / H} & \leqq \frac{|U W|_{G}}{|W|_{H}}
\end{aligned}
$$

It follows that

$$
\inf _{V \in \Re(G)} \frac{|K V|_{G}}{|V|_{G}} \leqq \frac{|K U W|_{G}}{|U W|_{G}} \leqq \frac{|\dot{K} U|_{G / H}}{|\dot{U}|_{G / H}}(I(H)+\epsilon) .
$$

Now $\Omega(G / H)=\{\dot{U} \mid U \in \Omega(G)\}$, so taking the infimum over $\dot{U}$ and then the supremum over $K \in \Re(G)$ we get $I(G) \leqq I(G / H)(I(H)+\epsilon)$. This proves Theorem 1.

CoRollary 1. Let $G=H_{0} \supset H_{1} \supset \cdots \supset H_{n-1} \supset H_{n}=E$ be a normal series of $G$. If $I\left(H_{i} / H_{i+1}\right)<\infty$ for $i=0, \cdots, n-1$, then $I(G)<\infty$. If $I\left(H_{i} / H_{i+1}\right)=1$ for $i=0, \cdots, n-1$, then $I(G)=1$.

COROLlaRy 2. If $G$ has a finite normal series with compact or abelian factors, then $I(G)=1$.

Corollary 3. If $G$ is as in Corollary 2, every p-admissible positive Radon measure is finite. If in this case, $\mathfrak{R}^{p}(G)$ is closed under convolution for some $p>1$, then $G$ is compact.

In [2] a measure $\mu$ was called $p$-admissible, if $\mu * \ell^{p} \subset \ell^{p}$.

The next corollary states the main result of [5].

Corollary 4. If $G$ is solvable, and $\mathfrak{R}^{p}(G)$ is closed under convolution for some $p>1$, then $G$ is compact.

The next two theorems are especially useful for discrete groups. 
THEOREM 2. If $H$ is an open subgroup of $G$, then $I(H) \leqq I(G)$.

Proof. Let the Haar measures in $G$ and $H$ be normalized, so that $|X|_{G}=|X|_{H}$ for $X \subset H$. Let $\Delta(x)$ be the modular function of $G$. Then for $M$ measurable in $H$ and $x \in G$, we have: $|M x|_{G}=\Delta(x)|M|_{G}$ $=\Delta(x)|M|_{H}$. Now, if $U \in \Re(G)$, there exist $x_{1}, \cdots, x_{r} \in G$ and $U_{1}, \cdots, U_{r} \in \Omega(H)$ such that $U=\bigcup_{1}^{r} U_{i} x_{i}$. For $K \in \Omega(H)$ it follows that $K U=U_{1}^{r} K U_{i} x_{i}$, the union being disjoint. Hence

$$
\begin{aligned}
\frac{|K U|_{G}}{|U|_{G}} & =\frac{\sum_{i=1}^{r}\left|K U_{i} x_{i}\right|_{G}}{\sum_{i=1}^{r}\left|U_{i} x_{i}\right|_{G}}=\frac{\sum_{i=1}^{r} \Delta\left(x_{i}\right)\left|K U_{i}\right|_{H}}{\sum_{i=1}^{r} \Delta\left(x_{i}\right)\left|U_{i}\right|_{H}} \\
& \geqq \min _{i} \frac{\Delta\left(x_{i}\right)\left|K U_{i}\right|_{H}}{\Delta\left(x_{i}\right)\left|U_{i}\right|_{H}} \geqq \inf _{V \in \Re(H)} \frac{|K V|_{H}}{|V|_{H}},
\end{aligned}
$$

and finally

$$
I(G) \geqq \sup _{K \in \Omega(H)} \inf _{U \in \Omega(G)} \frac{|K U|_{G}}{|U|_{G}} \geqq \sup _{K \in \Omega(H)} \inf _{V \in \Omega(H)} \frac{|K V|_{H}}{|V|_{H}}=I(H) .
$$

THEOREM 3. Let $\Omega$ be the system of all open, compactly generated subgroups of $G$. Then

$$
I(G)=\sup _{H \in \Omega} I(H) .
$$

Proof. From Theorem 2 it follows that $J=\sup _{\Omega} I(H) \leqq I(G)$. If $K \in \Omega(G)$, there exists $H \in \Omega$ with $K \subset H$ and

$$
\inf _{\Re(G)} \frac{|K U|_{G}}{|U|_{G}} \leqq \inf _{\Omega(H)} \frac{|K V|_{H}}{|V|_{H}} \leqq I(H) \leqq J,
$$

and hence $I(G) \leqq J, I(G)=J$.

Corollary 5. If $G$ is abelian, then $I(G)=1$.

This was proved in [2]. It also follows easily from Theorems 1 and 3.

Corollary 6. Let $G$ be discrete and $\Phi$ the family of all finitely generated subgroups of $G$. Then $I(G)=\sup _{\Phi} I(H)$.

Corollary 7. Let $G$ be discrete and $\Psi$ a family of subgroups such that (1) for $A, B \in \Psi$ there exists $C \in \Psi$ with $A, B \subset C,(2) G \subset=\bigcup_{H \in \Psi} H$. Then $I(G)=\sup _{\Psi} I(H)$.

This follows from the previous corollary. 
Corollary 8. $I(G)=1$ if $G$ is discrete and locally finite.

Corollary 9. Suppose that $G$ is discrete and contains a well-ordered chain $\Phi$ of subgroups $H_{\mu}$ such that:

(1) $H_{0}=E=\{e\}$, $e$ the identity in $G$;

(2) $H_{\mu}$ is normal in $H_{\mu+1}$;

(3) $H_{\lambda}=\bigcup_{\mu<\lambda} H_{\mu}$, if $\lambda$ is a limit ordinal;

(4) $G=H_{\omega}$ for an ordinal $\omega$;

(5) $H_{\mu+1} / H_{\mu}$ is either abelian or locally finite (more general: $\left.I\left(H_{\mu+1} / H_{\mu}\right)=1\right)$.

Then $I(G)=1$.

Proof. We use induction on $\omega$. If $\omega=0$, there is nothing to prove. Now assume Corollary 9 is true for ordinals less than $\omega$. Then especially $I\left(H_{\lambda}\right)=1$ if $\lambda<\omega$, hence Corollary 7 gives $I(G)=1$ if $\omega$ is a limit ordinal. Otherwise $\omega=\tau+1, I\left(H_{\tau}\right)=1, I\left(G / H_{\tau}\right)=1$ and so $I(G)$ $=1$, according to Corollary 1 .

We conclude with some remarks and a list of open problems.

1. Do there exist groups $G$ with $1<I(G)<\infty$ ? In [2] we mentioned that $I(G)=\infty$ if $G$ is the discrete free group with countably many free generators. Theorem 2 tells us that $I(G)=\infty$ if $G$ is discrete and contains a nonabelian free subgroup.

2. Let $H$ be a subgroup of $G$ such that $G / H$ is compact, but eventually without having an invariant measure. Is it still true that $I(G) \leqq I(H)$, or at least that $I(G)<\infty$ if $I(H)<\infty$ ?

3. Let $H$ be a discrete central subgroup of $G$. Is $I(G / H)=I(G)$ ?

So far as I can see, an affirmative answer to questions 2 and 3 would imply that every $G$ contains an open normal subgroup $H$ with $I(H)$ $=1$. It seems likely that there are also connections between the problems discussed here and the existence of invariant means on $C(G)$ and $\mathfrak{R}^{\infty}(G)$.

\section{REFERENCES}

1. N. Bourbaki, Integration, Chapter 7, Paris, 1963.

2. H. Leptin, Faltungen von Borelschen Maßen mit $L^{p}$-Funktionen auf lokal kompakten Gruppen, Math. Ann. 163 (1966), 111-117.

3. M. Rajagopalan, On the $L^{p}$-space of a locally compact group, Colloq. Math. 10 (1963), 49-52.

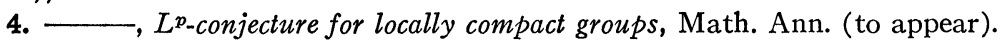

5. M. Rajagopalan and W. Żelazko, $L^{p}$-conjecture for solvable locally compact groups, J. Indian Math. Soc. 29 (1965), 87-92.

6. W. Żelazko, On the algebras $L_{p}$ of locally compact groups, Colloq. Math. 8 (1961), $115-120$.

7. —, A note on $L^{p}$-algebras, Colloq. Math. 10 (1963), 53-56.

University of Heidelberg, Germany 